\title{
Combination docetaxel (Taxotere), fluorouracil, and leucovorin (TFL), as first-line chemotherapy in advanced gastric cancer: a Hellenic Cooperative Oncology Group phase II study
}

\author{
Pavlos Papakostas $^{1}$, Dimitrios Tsavdaridis ${ }^{2}$, Paris Kosmidis ${ }^{3}$, Haralabos P. Kalofonos ${ }^{4}$, \\ Athanassios Sakantamis ${ }^{5}$, Dimitrios Janinis ${ }^{6}$, Dimosthenis Skarlos ${ }^{7}$, Dimitrios Bafaloukos ${ }^{8}$, \\ Aristotelis Bamias ${ }^{9}$, Nikos Xiros ${ }^{10}$, and George Fountzilas ${ }^{11}$ \\ ${ }^{1}$ Oncology Department, Hippokration General Hospital, 108, Vas.Sofias Avenue, Athens, Greece \\ ${ }^{2}$ IKA Hospital, Thessaloniki, Greece \\ ${ }^{3}$ Hygeia Hospital, Athens, Greece \\ ${ }^{4}$ Patras University Hospital, Patras, Greece \\ ${ }^{5}$ Hippokration Hospital, Thessaloniki, Greece \\ ${ }^{6}$ Agii Anargiri Cancer Hospital, Athens, Greece \\ ${ }^{7}$ Henry Dynan Hospital, Athens, Greece \\ ${ }^{8}$ Metropolitan Medical Center, Athens, Greece \\ ${ }^{9}$ Ioannina University Hospital, Ioannina, Greece \\ ${ }^{10}$ University General Hospital Attikon, Athens, Greece \\ ${ }^{11}$ Papageorgiou Hospital, Aristotle University of Thessaloniki School of Medicine, Thessaloniki, Greece
}

\begin{abstract}
Background. We assessed the efficacy and safety profile of a docetaxel (Taxotere; Sanofi-Aventis, France), fluorouracil (FU), and leucovorin (LV) combination (TFL), as first-line chemotherapy in patients with advanced gastric cancer.

Methods. Fifty-eight patients with advanced gastric cancer were entered in this phase II study. The chemotherapy regimen (TFL) was administered in an outpatient setting as follows: docetaxel $70 \mathrm{mg} / \mathrm{m}^{2}$ on day 1 and FU $500 \mathrm{mg} / \mathrm{m}^{2}$ and $\mathrm{LV}$ $300 \mathrm{mg} / \mathrm{m}^{2}$ on days 1 to 3 , every 3 weeks for six cycles.

Results. On an intent-to-treat basis, 4 complete (7\%) and 11 partial responses $(19 \%)$ were observed, with an objective overall response rate of $26 \%$; in addition, 22 patients $(38 \%)$ had stable and $15(26 \%)$ had progressive disease. For $6(10 \%)$ patients, response could not be evaluated. Responses were noted at all metastatic sites. With a median follow-up of 55 months, median survival was 9 months; median time to progression, 5.9 months; and median duration of response, 10 months. Toxicity was manageable and no toxic death was reported. Neutropenia was the most frequent severe toxicity and occurred in $30 \%$ of the patients. The main nonhematologic toxicities were alopecia $(76 \%)$, diarrhea $(30 \%)$, and stomatitis $(\mathbf{3 0} \%)$.

Conclusion. The results of this phase II study seem to indicate that the TFL regimen has moderate activity in patients with advanced gastric cancer, with acceptable toxicity.
\end{abstract}

Key words Docetaxel · Fluorouracil · Gastric cancer

Offprint requests to: $\mathrm{P}$. Papakostas

Received: September 12, 2005 / Accepted: November 17, 2005

\section{Introduction}

Worldwide, gastric cancer is the second most common cancer, following lung cancer [1]. Surgery offers the only possible cure. Although improvements in early diagnosis, preoperative assessment, and surgical technique have increased the number of curative resections over the past two decades, 5-year survival rates for all patients are in the range of only $10 \%-15 \%$ [2]. The poor outcome is due to the fact that $75 \%$ of patients are considered incurable at diagnosis (advanced disease), and moreover, even among patients with clinically resectable tumors, the relapse rate is high. Therefore, the use of chemotherapy in patients with gastric cancer has been a subject of great interest.

In advanced disease, the role of chemotherapy has been controversial, but recently a significant survival benefit for combination chemotherapy has been shown when compared with best supportive care [3].

The most widely used regimen was a combination of fluorouracil (FU), doxorubicin, and mitomycin (FAM), but this regimen provided questionable survival benefit compared to FU alone [4]. Over the past few years, several "second-generation" regimens of combination chemotherapy have been tested, but the survival obtained was not substantially better compared to that of previous regimens, whereas toxicity was more pronounced [5-7].

In an attempt to improve the response rate, and in particular, survival, in patients with advanced gastric 
cancer, new drugs and therapeutic interventions need to be tested.

Docetaxel (Taxotere; Sanofi-Aventis, France) is an antitumor agent belonging to the taxoid family. Docetaxel acts as an antimitotic agent, enhancing microtubule assembly and inhibiting the depolymerisation of tubulin, resulting in the inability of cells to divide. Docetaxel, as a single agent, appears to be effective in the treatment of gastric cancer. In two phase II studies $[8,9]$ monotherapy with docetaxel demonstrated the same response rate, of $24 \%$, and a median survival of 7.5 months.

On the other hand, a combination of FU and leucovorin (LV) has been found to be active in advanced gastric cancer [10], and the combination is a component of several "second-generation" regimens [5].

Therefore, we conducted a phase II study with a combination regimen of docetaxel, FU, and LV (TFL), in order to assess the efficacy of this regimen as first-line chemotherapy in patients with advanced gastric cancer. The dose and schedule of administration of FU and LV in the TFL regimen were identical to that used in the ELF (etoposide, LV, fluorouracil) regimen.

\section{Methods}

\section{Patient population}

From May 1997 to April 2001, 58 patients with advanced gastric cancer were entered in this study. Eligibility criteria included histologically proven unresectable or metastatic gastric adenocarcinoma, no prior chemotherapy or radiotherapy for metastatic disease, World Health Organization (WHO) performance status $0-2$, age 18 years or more, a life expectancy of at least 12 weeks, absence of a second primary malignant tumor (with the exception of nonmelanoma cutaneous carcinoma or cured intraepithelial cervical cancer), and no severe organ dysfunction, coronary insufficiency, or uncontrolled infections. Patients were also required to have bidimensionally measurable disease by computed tomography (CT) scans. Laboratory criteria included adequate bone marrow (absolute granulocyte count $\geq 1500 / \mathrm{dl}$ and platelets $\geq 100000 / \mathrm{dl}$ ), renal (serum creatinine levels $\leq 1.5 \mathrm{mg} / \mathrm{dl}$ ), and hepatic (serum bilirubin levels $\leq 1.5 \mathrm{mg} / \mathrm{dl}$ and aspartate aminotransferase [AST] or alanine aminotransferase [ALT] values $\leq 4$ times the upper normal limits) function. Informed consent was obtained from each patient according to institutional policy.

\section{Patient evaluation}

Pretreatment evaluation included a complete medical history and physical examination; complete blood cell counts (CBCs) with differential; a standard biochemical profile, as well as serum levels of carcinoembryonic antigen (CEA) and carbohydrate antiger (CA) 19.9; chest $\mathrm{X}$-rays; and CT scans of the chest and abdomen. Additional studies were performed as indicated. CBCs with differential were repeated on the day of treatment or if there was mucositis or fever.

In patients with grade 3 or 4 neutropenia, febrile neutropenia, or grade 4 thrombocytopenia, an absolute neutrophil count (ANC) was performed daily until the value was $1000 / \mathrm{dl}$ or more and platelets were $50000 / \mathrm{dl}$ or more.

A detailed medical history was taken and a complete physical examination was performed before each course of treatment to document symptoms of disease and the toxicities of chemotherapy. Biochemical tests and electrocardiograms were performed every 3 weeks. A clinical neurologic evaluation was performed in each cycle, and motor and sensory nerve-conduction velocity tests were performed in patients with 3 or 4 neurotoxicity. Lesions were evaluated at each cycle if they were assessable by physical examination. In all patients, response to treatment was assessed by physical examination, if appropriate, and by imaging procedures (CT scans) every three chemotherapeutic cycles. After the end of the treatment, the disease was evaluated by CT scans and tumor markers, every 3 months.

\section{Treatment}

The chemotherapeutic regimen (TFL) was administered as follows: docetaxel $70 \mathrm{mg} / \mathrm{m}^{2}$ on day 1 , as a 1 -h infusion; LV $300 \mathrm{mg} / \mathrm{m}^{2}$ on days 1,2 , and 3 , as a $2-\mathrm{h}$ infusion; and bolus FU $500 \mathrm{mg} / \mathrm{m}^{2}$ on days 1, 2, and 3 at mid-infusion of LV. The treatment was repeated every 3 weeks. All patients were premedicated with dexamethasone $8 \mathrm{mg}$, IV 12 and $6 \mathrm{~h}$ before the administration of docetaxel, in order to minimize the risk of hypersensitivity reactions and fluid retention syndrome. Moreover, dexamethasone ( $4 \mathrm{mg}$ orally every $12 \mathrm{~h}$ for four doses) was given after the chemotherapy. Cimetidine and antiemetics were also given.

The treatment was given in an outpatient setting. Dose adjustment was performed according to toxicity. A $25 \%$ dose reduction of TFL (docetaxel and fluorouracil) was required in patients with febrile or grade 4 neutropenia or grade 4 diarrhea. In patients with grade 3 diarrhea, a $25 \%$ dose reduction of FU was performed first and if, despite this reduction, grade 3 diarrhea occurred again, the dose of docetaxel was also reduced by $25 \%$. Severe diarrhea was managed with loperamide and ciprofloxacin administration.

Therapy was to be continued for six cycles, or until disease progression, consent withdrawal, or unacceptable toxicity, despite dose reductions, whichever hap- 
pened first. In patients with a complete response (CR), there was an attempt to deliver two more cycles of chemotherapy.

\section{Treatment evaluation}

All responses were defined according to WHO guidelines as follows: $\mathrm{CR}$, disappearance of all measurable or evaluable disease, with no new lesions; partial response (PR), 50\% reduction in the sum of the products of the largest perpendicular diameters of all measurable disease sites, with no new lesions; stable disease (SD), less than $50 \%$ reduction, or an increase of less than $25 \%$ of measurable disease, determined as stated above, with no new lesions; and progressive disease (PD), more than $25 \%$ increase in any measurable disease site or the appearance of new lesions. All responses were required to be sustained for at least 4 weeks. All toxicities were graded according to the WHO criteria.

\section{Statistical design}

The primary endpoint of this study was to assess the response rate, and secondary endpoints were the evaluation of toxicity, time to progression (TTP), and survival. The sample size was based on overall response rate (ORR). According to Simon's two-stage optimal design, assuming that the expected ORR would be at least $25 \%$ and the minimum acceptable response rate $10 \%$, a sample of 21 patients would be required in the first step. If a minimum of four responses were observed, a total of 55 patients would be accrued. Thereby, if at least ten responses occurred, the probability of accepting a treatment with a real response rate of less than $10 \%$ would be $5 \%$. On the other hand, the risk of rejecting a treatment (at the second stage) with a response rate of more than $25 \%$ would be $10 \%$.

All patients were included in the analysis for response and toxicity on an "intent-to-treat" basis.

Time to disease progression (TTP) was calculated from the initiation of treatment to the date that progression of the disease was documented (patients who discontinued their treatment for any reason or who died of what was regarded as disease-related causes were considered, at that time, as having disease progression). Survival was calculated from the initiation of the treatment to the date of last contact or to the date of death. Response duration was calculated from the date that the CR criteria were first met to the date that PD was first noted for complete responders, and from the date of the initiation of the treatment to the date that PD was first noted for partial responders. All patients without a corresponding event by the time of the analysis were considered censored. The Kaplan-Meier method was
Table 1. Patient characteristics

\begin{tabular}{lcc}
\hline$n$ & 58 & Percentage \\
\hline Age (years) & & \\
Median (range) & $63(33-81)$ & \\
Sex & & \\
$\quad$ Male & 40 & 69 \\
$\quad$ Female & 18 & 31 \\
Performance status ${ }^{\text {a }}$ & & \\
0 & 22 & 38 \\
1 & 26 & 45 \\
2 & 9 & 15 \\
$\quad$ Unknown & 1 & 2 \\
Prior adjuvant chemotherapy & 7 & 12 \\
Site of disease & & \\
$\quad$ Locoregional & 10 & 17 \\
$\quad$ Distant & 38 & 66 \\
$\quad$ Locoregional + distant & 10 & 17 \\
Number of metastatic sites & & \\
1 & 29 & 50 \\
2 & 23 & 10 \\
$\quad 33$ & 6 & \\
\hline
\end{tabular}

Values were rounded upward

${ }^{a}$ WHO criteria

used to calculate TTP and survival curves, and exact confidence intervals (CIs) were used to determine the $95 \%$ upper and lower confidence limits of the response rate (RR).

\section{Results}

Between May 1997 and April 2001, 58 patients entered this phase II study. Patient characteristics are listed in Table 1 . There were 40 men and 18 women, with a median age of 63 years (range, 33-81 years) and median WHO performance status of 1 (range, $0-2$ ).

\section{Best response and survival}

In six patients (10\%), response could not be evaluated, because two of them died prior to the first evaluation and four discontinued treatment (one patient due to unacceptable toxicity, two because they moved to another hospital, and one who refused to follow the treatment regimen).

On an intent-to-treat basis, 15 patients responded (26\%; 95\% CI, 15\%-39\%), 4 (7\%; 95\% CI, 2\%-17\%) with complete and 11 (19\%; 95\% CI, 10\%-31\%) with partial responses. Moreover, 22 patients (38\%; 95\% CI, $25.5 \%-52 \%$ ) demonstrated stabilization of the disease and 15 (26\%; $95 \%$ CI, $15 \%-39 \%)$ had progression of the disease. Median duration of response was 10 months (range, 3 to 28.4 months). Responses were observed at all metastatic sites. 


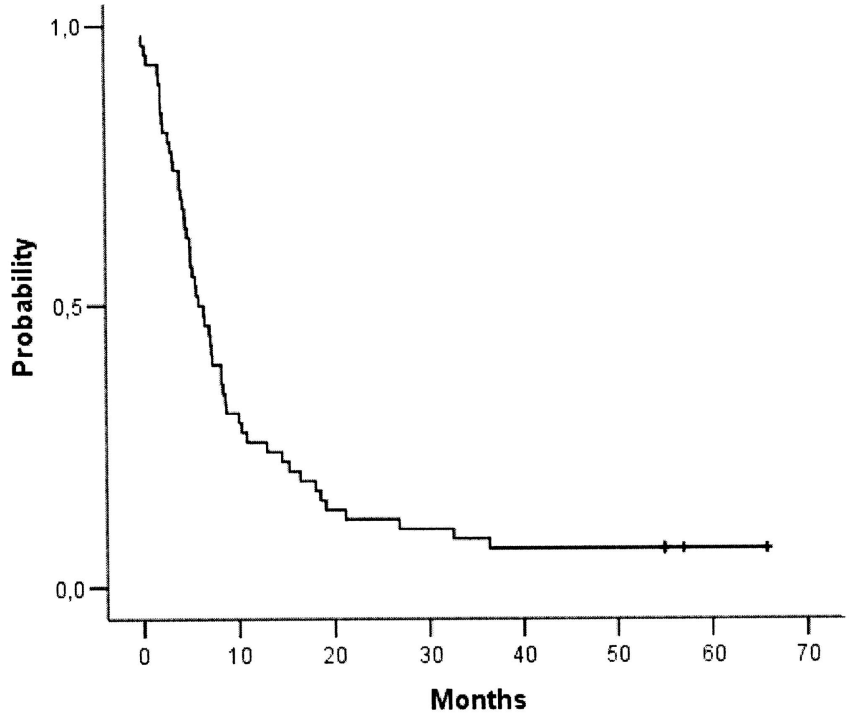

Fig. 1. Time to disease progression (TTP)

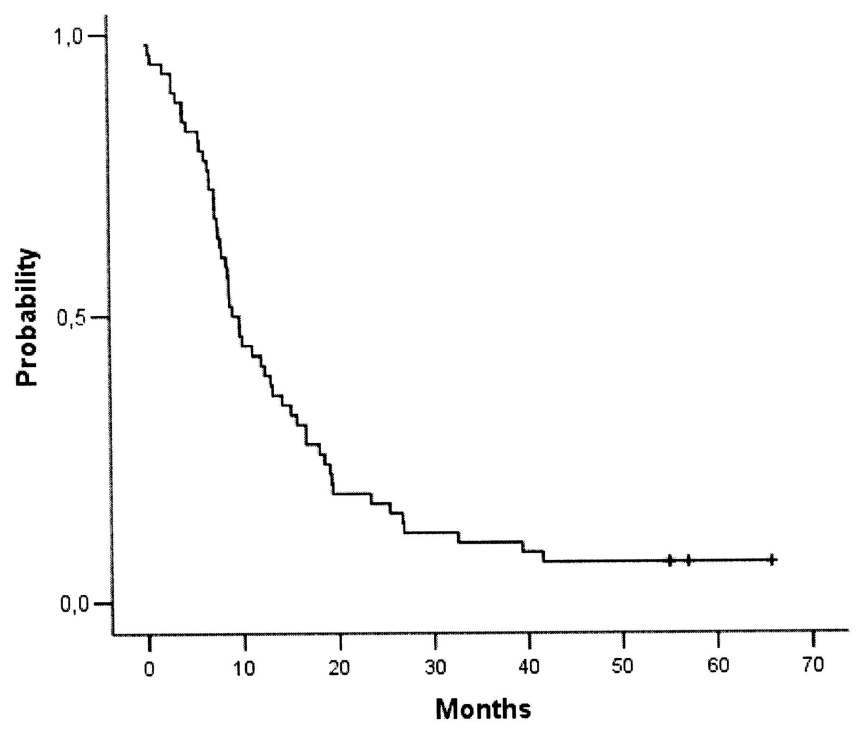

Fig. 2. Survival

After a median follow-up of 55 months (range, 0.01 to 65.7 months), 54 (93\%) patients had progressed and 4 (7\%) had died. Median TTP was 5.9 months (range, 0.01 to 65.7 months; Fig. 1), whereas median survival was 9 months (range, 0.01 to 65.7 months; Fig. 2).

\section{Treatment compliance and toxicity}

A total of 307 cycles of chemotherapy were delivered, $62 \%$ of them at full dose. The median number of chemotherapeutic cycles was 6 (range, 1 to 9 cycles) and the
Table 2. Incidence (\%) of various types of toxicity

\begin{tabular}{lrrrr}
\hline & \multicolumn{4}{c}{ Grade $^{\mathrm{a}}$} \\
\cline { 2 - 5 } Toxicity & \multicolumn{1}{c}{1} & 2 & 3 & 4 \\
\hline Neutropenia & 4 & 4 & 9 & 21 \\
Febrile neutropenia & - & - & 21 & - \\
Leukopenia & 4 & 2 & 11 & 5 \\
Thrombocytopenia & - & 2 & 2 & 2 \\
Anemia & 19 & 11 & 4 & - \\
Nausea-vomiting & 32 & 7 & 2 & - \\
Stomatitis & 12 & 14 & 2 & 2 \\
Diarrhea & 12 & 9 & 9 & - \\
Alopecia & 4 & 16 & 56 & - \\
Neuropathy & 9 & 4 & - & - \\
HSR & 5 & - & - & - \\
Myalgia/arthralgia & 9 & - & - & - \\
Fatigue & 14 & 4 & - & - \\
Fever & 2 & 11 & - & - \\
\hline
\end{tabular}

HSR, Hypersensitivity reactions, including rash, allergic reactions, peripheral edema, conjunctivitis

${ }^{\text {a }}$ WHO criteria

median interval between cycles was 21 days (range, 11 to 42 days).

The median dose intensity (DI) for FU was $410 \mathrm{mg} / \mathrm{m}^{2}$ per week (range, 132 to $521 \mathrm{mg} / \mathrm{m}^{2}$ per week) and for docetaxel, $22 \mathrm{mg} / \mathrm{m}^{2}$ per week (range, 8 to $29 \mathrm{mg} / \mathrm{m}^{2}$ per week). Median relative dose intensity for FU and docetaxel was 0.82 and 0.88 , respectively.

The incidence of major adverse events, shown as the maximum grade seen per patient, is listed in Table 2. No toxic death was reported.

Toxicity was the reason for treatment discontinuation in one patient. Neutropenia was the most frequent severe toxicity, and occurred in $30 \%$ of the patients $(9 \%$ grade 3 and $21 \%$ grade 4 ).

Fifty-nine percent of the patients received granulocyte-colony stimulating factor (G-CSF), either because of neutropenia or prophylactically. Grade 3/4 anemia and thrombocytopenia were seen in $4 \%$ of patients, whereas $11 \%$ of the patients received red blood cell and $4 \%$ received platelet transfusions, respectively.

\section{Discussion}

Despite its decreased incidence in developed countries, gastric cancer continues to be a significant worldwide health problem and therefore, its treatment remains a great challenge. Several combination regimens have been tested over the past few years, including EAP (etoposide, doxorubicin [Adriamyan], cisplatin), FAMTX, (fluorouracil, doxorubicin, methotrexate, LV), ELF, and ECF (epirubicin, cisplatin, fluorouracil). Although, all these "second - generation" regimens 
showed high ORRs, in the range of $40 \%$ to $50 \%$, in phase II trials, survival was only 7 to 11 months, not substantially better than that of "first - generation" regimens [5]. Moreover, in a few multicenter phase III trials conducted in the past few years using these regimens, lower ORRs and shorter median survival were achieved compared to the results of phase II trials [6,7]. The Japan Clinical Oncology Group (JCOG) conducted a randomized phase III trial that compared 5-FU alone with 5-FU+cisplatin (JCOG 9205). The primary endpoint of that study was overall survival, and both regimens showed equivalence not only in median survival time (approximately 7 months) but also in 1and 2 -year survival rates $(28 \%$ vs $29 \%$, and $7 \%$ vs $7 \%$, respectively). The toxicities were significantly lower with 5-FU alone than with 5-FU+cisplatin [11].

Therefore, the question of which regimen should be considered as "standard" chemotherapy for advanced gastric cancer cannot be answered at present.

The European Organization for Research and Treatment of Cancer (EORTC) study [12], which compared FAMTX with FAM, was the first trial demonstrating a survival advantage between chemotherapy regimens (in favor of FAMTX). In another randomized trial [7], which compared FAMTX with EAP, the ORR (33\% vs $20 \%$ ) and median survival (7.3 vs 6.1 months) were similar for both regimens, but the FAMTX regimen was significantly less toxic. More recently, a study from Royal Marsden [6] showed a superiority of ECF over FAMTX in ORR (45\% vs $21 \%$ ) and survival (9 vs 6 months). Finally, an EORTC randomized trial, which compared three combinations: FAMTX vs ELF vs cisplatin plus FU, showed that the RRs were approximately $25 \%$ for each combination, and none had a statistically significant impact on survival [13].

Therefore, no combination has emerged as a standard treatment for advanced gastric cancer. New drugs and therapeutic interventions need to be tested to improve RRs and, in particular, survival in patients with advanced gastric cancer.

In the present phase II trial we tested the TFL regimen, a combination of docetaxel with $\mathrm{FU}$ and $\mathrm{LV}$, in patients with advanced gastric cancer. To our knowledge, this is the first study reporting on the activity of this regimen, as first-line chemotherapy, in advanced gastric cancer. An objective ORR of $26 \%$ was achieved, with a median duration of response of 10 months.

As previously stated, the dose and schedule of FU and LV in the present study were the same as those in the ELF regimen. However, the addition of FU and LV did not increase the activity of the combination compared to that reported in the literature with docetaxel monotherapy $[8,9]$.

Furthermore, the $26 \%$ response rate achieved by the TFL combination in our study can be considered small compared to that reported recently with other docetaxel-based regimens, such as docetaxel plus FU continuous infusion (RR, 44\%) [14] and docetaxel plus cisplatin (RR, 53\%) [15]. A recently reported final analysis of a phase III study, which compared the triplet combination of docetaxel-cisplatin-FU versus cisplatin-FU, showed a statistically significant outcome in favor of the three-drug regimen, in terms of overall RR ( $37 \%$ vs $25 \%$ ), median TTP (5.6 vs 3.7 months), and overall survival (2-year survival was $18 \%$ vs $9 \%$ months) [16].

In our study, treatment compliance was satisfactory, because the median DI was more than $83 \%$ of the planned dose and treatment could be delivered on time. No toxic death was reported, even though the most frequent severe toxicity was neutropenia. The results of this phase II study seem to indicate that the TFL regimen is moderately effective in patients with advanced gastric cancer, with acceptable toxicity. In retrospect, the addition of bolus FU and LV to docetaxel did not appear to increase the activity of the combination compared to that of docetaxel monotherapy, and therefore, this regimen should not be explored in a phase III trial. On the contrary, docetaxel in combination with continuous infusion of FU, or with other active drugs such as cisplatin or irinotecan, should be further explored, in an attempt to improve the prognosis of patients with advanced gastric cancer.

\section{References}

1. World Health Report 1998: a vision for all. Geneva: World Health Organization; 1998.

2. Akoh AJ, McIntyre IMC. Improving survival in gastric cancer: review of 5-year survival rates in English-language publications from 1970. Br J Surg 1992;79:293-9.

3. Pyrhönen S, Kuitunen T, Nyandoto P, Kouri M. Randomised comparison of fluorouracil, epidoxorubicin and methotrexate (FEMTX) plus supportive care with supportive care alone in patients with non-resectable gastric cancer. Br J Cancer 1995;71: 587-91.

4. Cullinan SA, Moertel CG, Fleming TR, Rubin JR, Krook JE, Everson LK, et al. A comparison of three chemotherapeutic regimens in the treatment of advanced pancreatic and gastric carcinoma: fluorouracil vs fluorouracil and doxorubicin vs fluorouracil, doxorubicin and mitomycin. JAMA 1985;253:2061-7.

5. Wils J. The treatment of advanced gastric cancer. Semin Oncol 1996;23:397-406.

6. Webb A, Cunningham D, Scarffe JH, Harper P, Norman A, Joffe $\mathrm{JK}$, et al. Randomized trial comparing epirubicin, cisplatin and fluorouracil versus fluorouracil, doxorubicin and methotrexate in advanced esophagogastric cancer. J Clin Oncol 1997;15:2617.

7. Kelsen D, Atiq OT, Saltz L, Niedzwiecki D, Ginn D, Chapman D, et al. FAMTX versus etoposide, doxorubicin and cisplatin. A randomized trial in gastric cancer. J Clin Oncol 1992;10:541-8.

8. Sulkes A, Smyth J, Sessa C, Dirix LY, Vermorken JB, Kaye S, et al. Docetaxel (Taxotere) in advanced gastric cancer: results of a phase II clinical trial. EORTC Early Clinical Trials Group. Br J Cancer 1994;70:380-3. 
9. Mai M, Sakata Y, Kanamaru R, Kurihara M, Suminaga M, Ota J, et al. A late phase II clinical study of RP 56976 (docetaxel) in advanced or recurrent gastric cancer. Gan To Kagaku Ryoho (Jpn J Cancer Chemother) 1999;26:487-96.

10. Louvet C, de Gramont A, Demuynck B, Nordlinger B, Maisani JE, Lagadec B, et al. High-dose folinic acid, 5-fluorouracil bolus and continuous infusion in poor-prognosis patients with advanced measurable gastric cancer. Ann Oncol 1991;2:231-3.

11. Takiuchi H, Goto M, Kawabe S, Ohta S, Katsu K, et al. Secondline chemotherapy in gastric cancer (in Japanese). Gan To Kagaku Ryoho (Jpn J Cancer Chemother) 2005;32:19-23.

12. Wils JA, Klein HO, Wagener DJ, Bleiberg H, Reis H, Korsten F, et al. Sequential high-dose methotrexate and fluorouracil combined with doxorubicin: a step ahead in the treatment of gastric cancer: a trial of the EORTC Gastrointestinal Tract Co-operative Group. J Clin Oncol 1991;9:827-31.

13. Vanhoefer U, Rougier Ph, Wilke H, Ducreux MP, Lacave AJ, Van Cutsem E, et al. Final results of a randomized phase III trial of sequential high-dose methotrexate, fluorouracil and doxorubi- cin versus etoposide, leucovorin and fluorouracil versus infusional fluorouracil and cisplatin in advanced gastric cancer: a trial of the EORTC Gastrointestinal Tract Cancer Cooperative Group. J Clin Oncol 2000;18:2648-57.

14. Thuss-Patience PC, Kretzschmar A, Repp M, Kingreen D, Hennesser D, Micheel S, et al. Docetaxel and continuous-infusion fluorouracil versus epirubicin, cisplatin, and fluorouracil for advanced gastric adenocarcinoma: a randomized phase II study. J Clin Oncol 2005;23:494-501.

15. Roth AD, Maibach R, Martinelli G, Fazio N, Aapro MS, Pagani O, et al. Docetaxel (Taxotere)-cisplatin (TC): an effective drug combination in gastric carcinoma. Ann Oncol 2000;11:3016.

16. Moiseyenko V, Ajani JA, Tjulandin S, Majlis A, Constenla M, Boni C, et al. Final results of a randomized controlled phase III trial (TAX 325) comparing docetaxel (D) combined with cisplatin (C) and 5-fluorouracil (F) to CF in patients with metastatic gastric carcinoma (MGC) (abstract). Proc Am Soc Clin Oncol 2005;22: 4004. 